\title{
Maitriser les rythmes quotidiens : nouvelle revendication du droit à la ville?
}

Analyse des temps et espaces ralentisseurs, points d'ancrages et synchronisateurs des échelles et rythmes de vie métropolitains

Jean Grosbellet

\section{(2) OpenEdition}

Journals

Édition électronique

URL : http://journals.openedition.org/cdg/295

DOI : $10.4000 /$ cdg. 295

ISSN : 2107-7266

Éditeur

UMR 245 - CESSMA

Référence électronique

Jean Grosbellet, «Maitriser les rythmes quotidiens : nouvelle revendication du droit à la ville ? ",

Carnets de géographes [En ligne], 8 | 2015, mis en ligne le 01 septembre 2015, consulté le 20 avril 2019.

URL : http://journals.openedition.org/cdg/295; DOI : 10.4000/cdg.295

Ce document a été généré automatiquement le 20 avril 2019

\section{c) $(1)(9$}

La revue Carnets de géographes est mise à disposition selon les termes de la Licence Creative Commons Attribution - Pas d'Utilisation Commerciale - Pas de Modification 4.0 International. 


\section{Maitriser les rythmes quotidiens : nouvelle revendication du droit à la ville?}

Analyse des temps et espaces ralentisseurs, points d'ancrages et synchronisateurs des échelles et rythmes de vie métropolitains

Jean Grosbellet

\section{Introduction}

1 L'organisation temporelle des villes est directement engagée dans un processus d'accélération et de changements rapides dont plusieurs symptômes, comme l'éclatement des temporalités fordistes et l'accroissement du travail en dehors des horaires usuels, vers une plus grande flexibilité, sont déjà bien visibles (Rosa, 2010). La remise en cause des rythmes fordistes qui encadraient la vie urbaine au profit d'un modèle aux temporalités individuelles fractionnées et qui désagrègent les rythmes collectifs semble bien en place. L'évolution et l'accélération des modes de déplacements, de communication, des modes de vie et de cette «irrégularité comme caractéristique nouvelle de la vie urbaine bouleversant le schéma habituel métro-boulot-dodo" (Ascher, 1997), réinterrogent les cadres spatio-temporels de la ville. D'un côté, les temps et rythmes urbains sont de plus en plus souvent vécus et gérés individuellement et de l'autre les temps sociaux et collectifs qui structuraient la vie urbaine se sont estompés, laissant la place à une accélération des rythmes de vie sans repère, ni cadre affirmé. En ce sens, il semble impossible de penser la réappropriation de l'espace sans imaginer dans le même temps une réappropriation des rythmes de vie. Pour autant, la régulation de ces rythmes quotidiens est-elle devenue un nouveau support de revendication du droit à la ville (Lefebvre, 1968) ? «Ralentir » est-il devenu un nouvel enjeu des luttes urbaines face à l'immédiateté des logiques financières, l'impératif de compétitivité et l'accélération sociale du temps qui façonnent la ville néolibérale ? Ce retour au concept de «droit à la 
ville " semble traduire «une prise de conscience de l'accélération et de la généralisation des formes d'injustices dans la ville induites par le néolibéralisme. » (Costes, 2014).

2 Ce n'est donc pas un hasard si les questions temporelles font l'actualité politique en France notamment avec le débat sur le travail du dimanche, la réforme des rythmes scolaires, l'extension des horaires d'ouverture des services publics et des transports en particulier. Les politiques urbaines et l'économie marchande ne cessent d'augmenter la vitesse des flux de personnes, de données, de marchandises et cela de manière continue, 24 heures sur 24, 365 jours par an. La fluidification des temps sociaux souhaitée par la classe politique, à travers le développement de la vitesse et de l'instantanéité, semble offrir aux habitants une meilleure maîtrise de leur rythme de vie. Ils ont de nouvelles possibilités de parcours urbains et temporels, en accédant à plus de services en moins de temps, comme une sorte de ville à la carte, rendant possible des combinaisons. Mais ces nouveaux usages de la ville sont aussi socialement inégaux et différenciés (Ascher, 1997). Un certain nombre d'individus restent en marge des processus temporels: ils ne participent ni au mouvement d'émancipation individuelle, ni au mouvement de redécouverte des nouvelles temporalités collectives. Comment redonner à ces individus des possibilités de régulation de leurs parcours quotidiens ? La ville est-elle condamnée à produire des désynchronisations acceptées, sources de fragilités et d'inégalités? Ou appelle-t-elle d'autres conjugaisons des temps multiples, d'autres concordances qui inventent les rythmes propres à un groupe, à une société, à un territoire ? Chaque citadin évolue à des rythmes différents au sein de son espace, parce qu'il maitrise mieux son temps qu'un autre. Mais si le rapport aux phénomènes d'accélération et de ralentissement relève de conceptions individualistes, la notion de lenteur possède à la fois une définition propre à chaque individu mais aussi -et c'est là son intérêt politiqueune définition collective. Elle fait ici figure de cadre, de repère structurant car identifiable et partageable par le plus grand nombre.

\section{Définitions et hypothèses de travail}

3 La lenteur renvoie à un état, une stabilité, contrairement aux mouvements que constituent l'accélération et le ralentissement. Elle correspond à des temporalités singulières et cycliques, celles des temps collectifs et sociaux au sein desquels s'accordent et se synchronisent les temps individuels. Ces temps sociaux agissent comme des marqueurs à l'échelle du quotidien et contribuent à donner un rythme propre à chaque ville, à chaque territoire. C'est par rapport à ces temps collectifs que les individus ralentissent et accélèrent leurs temps quotidiens. Il convient donc de repositionner la lenteur par rapport aux processus d'accélération et de ralentissement au sein des villes. Ralentir renverrait plutôt à une action individuelle qui se manifeste par une rupture dans le rythme linéaire et l'enchaînement des temps quotidiens.

En lien avec sa thèse sur l'accélération sociale du temps, Harmut Rosa identifie trois types de ralentissements qui marquent des ruptures dans les processus d'accélération: les ralentissements naturels, physiques, biologiques ou anthropologiques qui sont partagés par tous; les ralentissements dysfonctionnels, qui représentent des contrecoups non intentionnels au processus d'accélération, les embouteillages ou le burn-out par exemple, et les ralentissements idéologiques, comme les mouvements slow par exemple, qui réagissent aux effets écologiques et sociaux déstructurants de l'accélération. 
5 Face aux phénomènes d'accélération sociale (speed dating, fast food, caisses express, siestes éclairs), l'homme a généré des mécanismes inverses, comme autant de réflexes de survie, par l'apparition de mouvements slow (slow-city, slow food) ou par des accroches politiques sur la ville durable, la ville lente, l'agriculture urbaine, les « villes où il fait bon vivre » en particulier.

6 En s'appuyant sur cette analyse, nous pouvons séparer d'un côté les ralentissements subis (naturels et dysfonctionnels) et de l'autre les ralentissements idéologiques qui semblent choisis. Le sentiment de ralentissement serait éprouvé dans le cadre d'une expérience choisie (rapide ou lente). A l'inverse, le sentiment d'accélération serait le résultat d'une expérience subie (rapide ou lente). Ces rapports subjectifs dépendraient de la capacité de chaque individu à maîtriser son temps, aux représentations du temps et aux valeurs attachées au couple rapidité-lenteur. Nous montrerons que la maîtrise du temps conduit au sentiment de ralentissement recherché mais que paradoxalement ce sentiment de lenteur peut être perçu dans un rythme de vie rapide, à condition qu'il soit maîtrisé. Quels sont alors les temps et espaces du quotidien qui génèrent un sentiment de ralentissement chez les habitants et usagers de la métropole?

7 Dans une première partie, nous présenterons les résultats d'une étude de spatialisation des emplois du temps de différents individus, réalisée entre février et décembre 2014. Elle comprend l'analyse des emplois du temps ainsi que des schémas types représentant les parcours quotidiens. A partir de ces résultats, nous verrons, dans une deuxième partie, qu'une meilleure maîtrise de leur temps quotidien passe par deux niveaux d'intervention : le premier sur les rythmes endogènes des parcours quotidiens à travers l'aménagement d'espaces ralentisseurs, sorte de «hubs » métropolitains, favorables aux synchronisations individuelles multi-vitesses; le second en replaçant leurs parcours individuels en interaction avec les rythmes exogènes et structurants des temps sociaux et naturels.

\section{L'analyse des emplois du temps : comprendre et représenter les rapports à l'espace-temps quotidien}

8 L'étude a pour but de montrer les différentes manières de parcourir le temps et l'espace dans la métropole bordelaise, terrain de mes recherches doctorales, via une analyse sociologique de type qualitative. Il s'agit d'étudier l'usage de la ville par le temps en spatialisant des modes de vie. L'analyse se fonde sur les pratiques quotidiennes des individus dans leur espace vécu et, plus particulièrement ici, entre lieu de domicile et lieu de travail (Frémont, 1999). Les personnes interrogées parcourent plusieurs lieux dans une même journée (travail, transports, vacances...) et dans la notion d'espace vécu, le trajet est aussi important que le lieu (Bourdin, 2005). Un premier niveau d'étude a porté sur une analyse du temps libre des personnes et de leur capacité à le mobiliser, à partir d'une observation de leur emploi du temps et d'un entretien semi-directif. Un second niveau d'analyse a montré l'intérêt de spatialiser l'espace vécu des parcours quotidiens des individus, leur territorialité et leurs appropriations. Cette étude donne à voir une image originale du milieu temporel de la métropole bordelaise, comme l'ensemble des temporalités à l'œuvre dans un même espace, tel que défini par William Grossin et dans lequel se développent et s'entrechoquent continuellement des phénomènes 
d'accélération et de ralentissement, subis ou choisis, que les groupes sociaux expérimentent afin d'organiser et de rythmer leur emploi du temps (Grossin, 1996).

Cette enquête porte sur un échantillon de 18 personnes qui vivent et/ou travaillent à environ une heure de transport de Bordeaux. Elles ont entre 25 et 34 ans. Cette classe d'âge, qu'utilise régulièrement l'INSEE, correspondrait à une période de "stabilisation et de confirmation des choix » (Percheron, 1988). On retrouve des profils très différents, de l'étudiant au jeune actif, d'une vie de célibataire à une vie de famille, d'un emploi stable à un emploi précaire. Les 25-34 ans reflètent un échantillon particulièrement riche pour étudier les vies intenses et mobiles d'une métropole dont ils composent, dans le cas bordelais, $30 \%$ de la population (INSEE, RP2011). L'objectif de l'étude n'est pas d'atteindre l'exhaustivité en matière de représentativité, mais bien d'éclairer un objet et de mesurer le caractère hétérogène des perceptions et pratiques temporelles de l'espace. Plusieurs acteurs aux profils différents ont été interrogés en fonction du sexe, du domicile, du type de ménage et de l'activité professionnelle. Nous avons approché autant de femmes que d'hommes, des personnes célibataires et des personnes vivant en couple, avec ou sans enfants.

Le facteur du genre est ici déterminant. La conciliation des temps sociaux est plus difficile pour les femmes, qui doivent jongler entre le temps professionnel (souvent à temps partiel), les tâches domestiques (même si une tendance à l'équilibre au sein du foyer est observée) et le temps "parental» (encore deux tiers de ce temps repose sur les femmes au sein du couple) (Barrère-Maurisson, Rivier, 2004 ; Cingolani, 2012). Les personnes les plus diplômées ont un revenu plus élevé et travaillent plus. Elles passent néanmoins plus de temps à réaliser des activités de loisir, leurs revenus permettant «d'acheter du temps ». A l'inverse, si les personnes moins diplômées disposent de plus de temps libre (en comptant l'effet du chômage et du temps partiel), leurs loisirs se déroulent le plus souvent à domicile (Coulangeon, Menger, Roharik, 2002). Une importance toute particulière a donc été accordée à la prise en compte des conditions sociales comme le révèle le tableau de synthèse ci-après. Ainsi, les revenus des enquêtés vont de 500 à 2200 euros net par mois, on retrouve également des personnes aux catégories socioprofessionnelles diverses.

Dans un premier temps, chaque enquêté a fait parvenir son emploi du temps de la semaine sur papier libre. La consigne était de représenter en colonnes les 5 premiers jours de la semaine. Cette représentation guidée n'était pas prévue à l'origine mais les premiers emplois du temps étaient trop différents et ne permettaient pas une analyse approfondie. Le week-end n'est pas étudié, nous avons souhaité porter notre attention sur une semaine restreinte afin de mieux en saisir les enjeux. Les semaines étudiées étaient des semaines « classiques » pour les acteurs, c'est-à-dire sans congés ou emploi du temps exceptionnel (déplacements, maladie, congés, vacances). Une fois les emplois du temps communiqués, nous observons deux grandes formes de représentation: la première par des blocs qui symbolisent souvent une macro-temporalité comme le temps de travail; et la seconde par des tirets qui représentent plutôt une liste d'activités secondaires et qui ne sont pas toujours clairement reliées à une heure donnée.

La semaine écoulée, un entretien est réalisé avec chaque personne enquêtée. Ces 18 entretiens, d' $1 \mathrm{~h} 30$ à $2 \mathrm{~h} 30$, représentent un matériau très riche. La première partie de l'entretien revient sur l'emploi du temps et sur les décalages éventuels avec la semaine vécue. Dans un deuxième temps, un entretien semi-directif cherche à définir les modalités d'organisation et de maîtrise de l'emploi du temps des individus en les 
interrogeant sur leur temps libre disponible et sur leur capacité à le mobiliser. L'entretien semi-directif individuel a été préféré au recueil de données par questionnaire car il permet de mieux connaître les justifications des acteurs quant aux logiques mises en œuvre dans leur parcours. Des allers-retours entre représentations sociales et pratiques sont nécessaires pour comprendre les parcours. Lors du troisième temps de la rencontre, nous spatialisons l'emploi du temps pour faire ressortir les parcours. Ces parcours nous servent ici à ressortir l'espace vécu par le biais des pratiques de mobilité quotidiennes plus ou moins fréquentes. Sur une photocopie de carte IGN, nous marquons pour chaque jour les lieux fréquentés et traçons les parcours quotidiens entre accélérations et ralentissements.

Figure 1. Profil des personnes interrogées

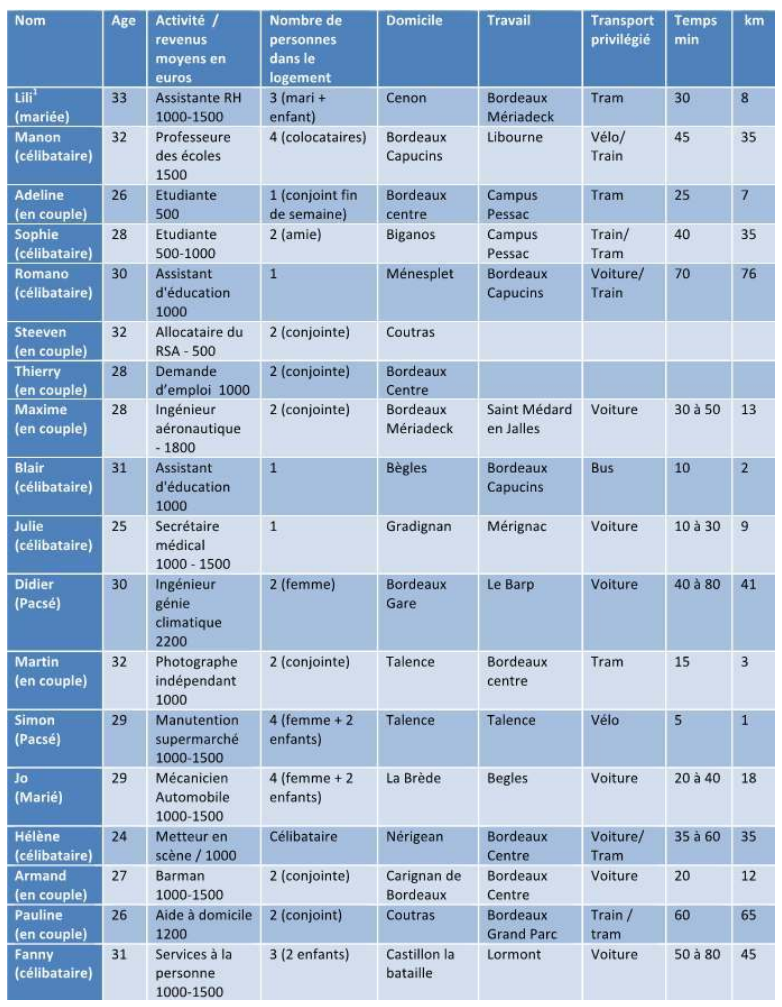

Source : J. Grosbellet, 2014 
Figure 2. Localisation du domicile et du lieu de travail des personnes interrogées

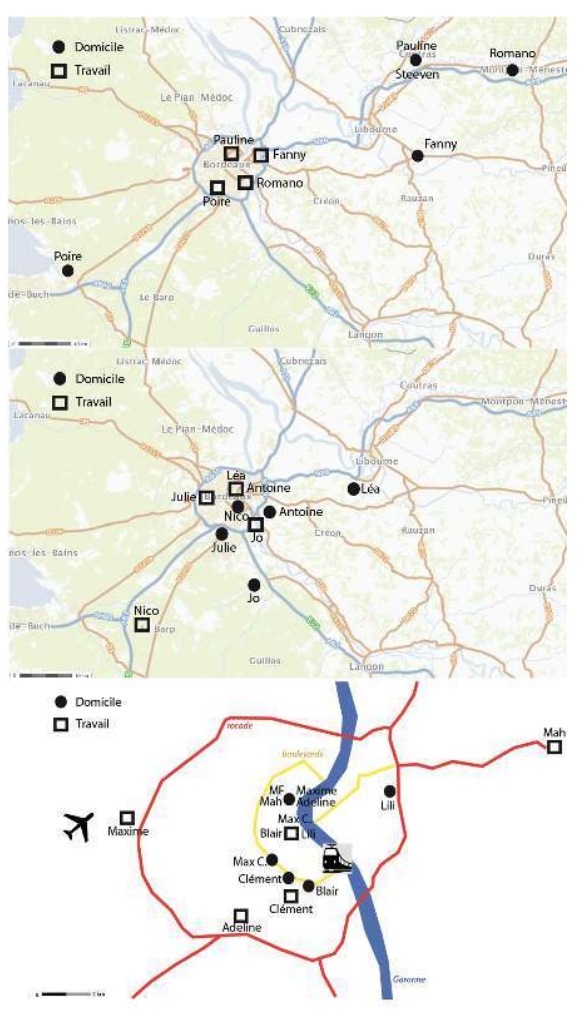

Source : J. Grosbellet 2014

\section{Equilibrer son emploi du temps : entre paradoxes et représentations socio-spatiales du couple vitesse/ lenteur}

Les premiers emplois du temps papiers analysés laissent apparaître dans le milieu temporel bordelais la présence de macro-temporalités. Issues des grandes fonctions urbaines, structurantes pour un ensemble d'individus, elles se caractérisent notamment par un début et une fin, ont des amplitudes importantes et occupent une grande partie de la semaine. Ainsi pour chacun, se déplacer, habiter, se récréer et travailler sont des macro-temporalités qui rythment le quotidien. Entre ces temps structurants, prennent place des temps de respiration, de pauses, qui ne sont pas notés sur le papier. Cependant, lors de la phase d'entretien, il est apparu qu'une multitude de micro-temporalités se développent dans ces espaces de respiration, de pause, mais aussi d'attente et d'ennui et se superposent même aux macro-temporalités.

La quasi-totalité des personnes considèrent avoir du temps libre dans une journée de travail. Mais ces mêmes personnes déclarent aussi avoir un rythme de vie trop rapide. Le temps libre ne servirait donc pas forcément à réguler ce rythme mais plutôt à le maintenir sous tension en multipliant les activités. Nous notons également deux points de convergence pour la majorité des sujets de l'enquête. Le temps libre, celui du choix, est le plus souvent vécu entre la sortie du travail et le moment de regagner son domicile (la pause déjeuner est également souvent citée). Le choix et la possibilité de mobiliser son 
temps sont nécessaires à la perception du temps libre au regard de son caractère éphémère. Pauline qui travaille comme aide à domicile à temps partiel explique :

C'est très frustrant car mes journées sont souvent pleines de trous. Donc j'ai du temps libre mais c'est surtout du temps d'attente. Au début, je trouvais ça cool, je me baladais en ville, je lisais dans des cafés, mais à la longue, ça revient cher et j'ai surtout envie de terminer la journée, pour rentrer chez moi. Rien que de penser au temps de trajet ça me déprime. L'hiver je viens en voiture, ça me fait un moment tranquille sur la route et je peux aller dans ma voiture quand je n'ai rien à faire. C'est ça le pire, je dois me dépêcher le matin pour pointer et après je me retrouve parfois à attendre deux heures toute seule entre deux prestations. Le soir, je veux juste que ça se finisse.

Pour Steeven, allocataire du RSA et qui vit à Coutras :

Techniquement, c'est vrai qu'en ce moment, j'ai pas mal de temps, mais je ne dirais pas du temps libre, plutôt du temps à rien faire. Il n'y a pas grand-chose dans le coin et puis je n'ai pas trop de thunes.

Alors que pour Thierry, demandeur d'emploi vivant à Bordeaux :

$\mathrm{Au}$ début, je culpabilisais beaucoup. Je voyais mes potes bosser, ma copine me mettre la pression et mes parents surtout. Mais avec le temps ça va mieux. Je vais prendre des cours de guitare, j'apprends l'espagnol, je vais au cinéma, je sors, je fais le marché, je cuisine, c'est vraiment cool. Je fais plus de trucs qu'avant, quand je bossais, mais je suis moins crevé.

Cette dernière comparaison montre bien la nécessité de pouvoir mobiliser son temps libre pour le rendre effectif par l'accès aux services mais aussi, dans ce cas précis, par le capital économique. Une autre explication émerge en transparence, celle de la représentation sociale de l'hyperactivité ou de la non-activité. Un rythme de vie soutenu confère une certaine valorisation sociale, un rythme lent est le plus souvent mal perçu et dévalorisant (Kaufmann, 1997 ; Honoré, 2005). Steeven avoue « rester discret la journée » et « avoir un peu honte quand le facteur passe ", "de lui ouvrir en pyjama ». A la voisine il dit "être étudiant " afin d'éviter les discutions sur sa situation. "En ville, c'est différent, il y a beaucoup de gens qui ne travaillent pas tout le temps. A la campagne, si tu fais des courses à $10 \mathrm{~h}$ du matin, tout le monde sait que tu n'as pas d'emploi. » Maxime, pour sa part, entrevoit ce phénomène lors de la pause déjeuner dans son entreprise :

$\mathrm{Au}$ boulot, les rapports entre les gens sont vraiment basés sur l'apparence et la compétition. La culture école quoi....Les gars s'inventent des vies overbookées. Ça donne un air important d'avoir du boulot par-dessus la tête. Le côté dynamique qui colle avec le terme de cadre ou de jeune actif... Avec leurs portables, les mecs envoient des mails de nuit, à $5 \mathrm{~h}$ du matin, et si tu n'es pas réactif, tu prends des petites remarques, à la longue c'est pénible. Le pire c'est à la pause déjeuner, quand tu pars manger et que tu croises ton boss ou un de tes collègues avec un sandwich qui te dit avec le sourire : bon appétit les gars, moi j'ai pas le temps, trop de taf, j'aimerais bien être à votre place... trainez pas trop quand même...

Armand qui travaille la nuit comme barman dans l'hyper-centre de Bordeaux ressent aussi ce phénomène :

Ce qui est assez compliqué à gérer c'est le regard des gens, quand ils te regardent le matin, tu sens qu'ils se disent que tu sors de soirée. Ou en journée quand les gens pensent que tu ne travailles pas. Ils ne supportent pas que tu ne sois pas speed, c'est comme une insulte pour eux.

L'analyse des emplois du temps reflète le caractère très personnel du rapport au temps et notamment au temps libre. Il appartient de plus en plus à chaque individu de produire son propre rythme. Les rythmes quotidiens sont de moins en moins régulés par les rythmes collectifs. Les personnes interrogées doivent tenter de s'adapter en permanence 
aux temporalités dans lesquelles elles évoluent et auxquelles elles sont confrontées, par des ajustements, des synchronisations, mais aussi des perturbations et des désynchronisations. Certains vont considérer le temps de transport comme un temps contraint, une lenteur subie, alors que d'autres vont trouver dans cet espace-temps un moment pour eux, une coupure salvatrice dans la journée. Pour Manon :

J'ai du temps pour moi le soir, après le boulot, dans le train de Libourne à Bordeaux, j'en profite pour bosser encore un peu. En arrivant à la gare, je sens vraiment que la journée est finie, même si j'ai encore un peu de vélo pour rentrer chez moi.

Parfois le même trajet est vécu différemment en fonction de l'humeur du jour. Toutefois, si le sentiment de ralentissement est subjectif, il semble que certains lieux et certains moments de la journée soient plus propices aux ralentissements. Ils correspondent notamment à des temps favorables à la maîtrise et à l'organisation de son emploi du temps. Un autre élément important transparaît des entretiens. Pour améliorer la maitrise de leur temps, nombreux sont les enquêtés à avoir fait allusion à leur smartphone comme dispositif d'aide à l'organisation, propice au sentiment de ralentissement. En recoupant les informations sur ce sujet, nous pouvons constater que l'usage du smartphone témoigne de la réactivité de l'emploi du temps du sujet. Dans le cas de Maxime, Martin ou encore Adeline, qui combinent maîtrise temporelle et centralité des lieux de vie, l'appropriation de la ville semble totale. On observe même une pratique plus diversifiée de la ville à l'échelle de la semaine, en termes d'activités différentes et de lieux fréquentés. La centralité, qui se déplace au fil de la journée selon les flux (Marzloff, 2005), combinée à la connectivité, leur permet de construire des parcours spatio-temporels en temps réel et d'avoir une meilleure maîtrise de leur temps et de leur espace. Martin donne un exemple :

Parfois en début de semaine j'ai l'impression qu'elle va être tranquille, que je n'ai rien de prévu et puis finalement, chaque jour, au fil des rencontres, des invitations Facebook, des mails, des coups de fils, des textos.... Je me retrouve avec 10000 trucs à faire. Un client, des gens à voir, des potes de passage, un concert, un vernissage... Je ne planifie pas vraiment ma vie du coup, c'est intense, mais je m'y retrouve. C'est plutôt avec ma compagne qui a des horaires « classiques » que c'est parfois un peu compliqué.

18 Avec le processus d'accélération sociale, le futur est compressé vers le présent (Dubar, 2008). Les phénomènes d'accélération sociale sont le plus souvent étudiés par la représentation tridimensionnelle du temps entre le temps du passé : la mémoire ; celui du présent: l'attention et celui du futur: l'attente (Augustin, 1962; Benveniste, 1966). Ce mécanisme de virtualisation, d'immédiateté du futur, a pour effets de diminuer l'attente, mais inscrit la société dans un présentisme angoissant (Chesneaux, 2004). «La réalité du temps a été remplacée par la publicité du temps » (Debord, 1967). Le futur semble incertain, nous ne nous projetons pas à long terme et les nouvelles technologies sont indispensables pour nous rassurer. Maxime dit

«...profiter d'être en voiture, dans les bouchons, pour envoyer des messages Facebook à des amis pour voir s'il y a quelque chose de prévu le soir même ... » tout en «... écoutant de la bonne musique pour se détendre ... » sans oublier de » ... régler deux trois trucs...des mails du boulot, que j'avais la flemme de traiter en partant... ».

19 Dans le même temps, ce présentisme semble rendre le passé plus lointain, nous oublions plus vite, à cause d'un trop grand nombre d'événements à traiter mais aussi parce que nous savons, inconsciemment peut-être, qu'Internet se substitue à nos mémoires. A partir de ce constat, ralentir passerait notamment par une remise à distance du futur, afin de se projeter vers un horizon des possibles, par la virtualité humaine, le rêve et l'imagination 
en particulier. Le présent se doit d'être ouvert sur l'avenir par le ressourcement dans le passé (Chesneaux, 1996), car "le culte de la vitesse est à la fois un progrès et une violence " (Virilio, 2003).

20 Cette réactivité correspond à une maîtrise forte de son temps. Pour certains enquêtés, le temps semble même se définir comme un capital déterminant dans l'organisation de leur quotidien et permettant aux individus d'obtenir des avantages sociaux, dans notre cas le ralentissement et le bien-être (Bourdieu, 1979). Au même titre que leur capital économique, social ou culturel, ils cherchent à " accumuler » du temps, à le " dépenser " et parfois en «manquent ». La ville se vit intensément et en temps réel. Les personnes qui ont un emploi du temps plus contraint semblent se servir de leur smartphone pour prévoir, anticiper. Ainsi, dans le cas de Julie qui habite en banlieue, elle dit :

«...je passe mon temps au travail sur Facebook à envoyer des messages à mes amis pour voir si je rentre directement chez moi après le travail ou si je vais en ville...»

Elle sait que « si je rentre chez moi, je ne ressortirai pas ».

Ainsi, son comportement va tendre à anticiper au maximum le futur, pour ne pas mettre en danger son équilibre spatio-temporel. Il est impossible pour elle de maintenir le futur à distance, d'imaginer se rendre en ville pour flâner, en espérant qu'il se passe quelque chose. Elle évoque en particulier un emploi du temps contraint, elle ne peut donc parier sur l'avenir :

J'ai besoin de faire une pause après le boulot mais il faut que je rentabilise au maximum mon temps. J'ai besoin de savoir exactement, où on va prendre un verre, histoire de ne pas tourner des heures en ville en voiture.

Les nouvelles technologies viennent ici en support pour une meilleure maîtrise de son temps afin de pallier un éloignement à la centralité. Paradoxalement, en accélérant le processus de décision, elle a le sentiment de gagner le temps de pouvoir prendre une pause et ainsi ralentir son agenda. Dans le cas de Pauline, son smartphone lui permet aussi, de façon paradoxale, d'accélérer l'attente subie en la comblant de micro-activités, mais aussi d'examiner les horaires de train, les correspondances, de vérifier l'adresse d'un client. Elle voit en son smartphone:

une aide précieuse si jamais il y a un problème dans la journée, une grève, une panne de tram, de train, c'est rassurant !.

Cette maîtrise relative contribue néanmoins au sentiment de ralentissement, celui d'avoir un peu de temps devant soi. L'utilisation du smartphone semble être à la fois pour certains individus la cause d'un sentiment d'accélération du temps en entretenant le multitasking (réalisation de plusieurs activités en même temps), en accumulant les informations ; mais aussi pour d'autres la solution pour mieux organiser et maîtriser son temps.

\section{Les parcours quotidiens, entre accélération et ralentissement : la recherche d'un équilibre spatio- temporel par la maîtrise de son emploi du temps}

Nous avons réalisé, à partir des cartes de parcours individuels, des schémas simplifiés donnant à voir des parcours types en fonction des personnes interrogées. Cette volonté de représenter les parcours spatio-temporels du quotidien peut s'inscrire en lien avec la Time-Geography (Hagerstrand, 1970) et plus particulièrement en tant que modèle méthodologique (Chardonnel, 1999). Ces typologies servent uniquement à l'analyse et à la 
compréhension de l'étude. Nous représentons ici spécifiquement le temps de «l'après travail ", majoritairement considéré par les enquêtés comme privilégié pour ralentir (le temps de la pause-déjeuner est également cité de nombreuses fois). Les parcours témoignent des capacités de chacun à choisir ou subir les temps ralentisseurs qui jalonnent leur quotidien. Nous parlerons de « hubs " pour identifier ces espaces dans leur globalité. Ceux-ci sont multiples, on retrouve des centralités urbaines, des centralités économiques (zones d'activités, zones commerciales), des lieux de connexions de transport en particulier. Leurs accès sont plus ou moins aisés en fonction du type de mobilité. Leurs offres de services sont très inégales, notamment dans le rapport entre services marchands et non marchands. La qualité paysagère et urbaine diffère également. Ils ont pour points communs de capter les flux d'habitants en concentrant des activités dans un même espace de proximité. Cela permet d'effectuer un grand nombre d'actions quotidiennes en peu de temps.

\section{Une centralité comme espace vécu : un espace favorable aux choix et au ralentissement individuel et collectif}

Le premier parcours type est celui d'un individu qui a une forte maîtrise de son temps. Le plus souvent son domicile et/ou son lieu de travail se trouvent dans un hub : centre-ville, zone commerciale, zone d'activité, en particulier. L'axe de transport domicile-travail n'est pas structurant, le hub permet de réguler son temps libre. Dans le cadre d'un centreville, le choix est maximal et de multiples combinaisons de parcours sont possibles. Blair qui vit et travaille dans le centre de Bordeaux :

je ne vois pas le trajet en vélo de 10 minutes tous les matins comme un temps de transport, c'est mon mode de déplacement pour tout et tout le temps. Le vélo c'est parfait, tout est accessible rapidement à Bordeaux. [...] Je vis et travaille dans le même quartier, quand je débauche, souvent je repasse par la maison, après j'avise en fonction de ce qui se dessine. [...]

Pour Adeline, en dehors du trajet en tramway pour l'université qui est «le moment le plus long dans la journée », une fois en ville, « je suis chez moi !», «j'ai toujours dix mille trucs à faire, des petites courses, prendre un verre, aller courir, simplement marcher sur les quais, je rentre et je sors en permanence.». Il se peut également que ce soit le lieu de travail qui joue le rôle de hub. On remarque souvent ce phénomène pendant le temps du déjeuner. Jo qui travaille dans une zone commerciale, à Bègles Rives d'Arcins, profite de cette situation :

Entre midi et deux, je mange dans les restaurants de rives d'Arcins, il m'arrive de faire les courses pour la maison aussi, l'été on se fait un casse-croûte et on va pêcher sur les étangs, c'est pratique, c'est tout proche. Il y a tout à cet endroit. Tu as de la place pour te garer, pleins de magasins différents, des bars si tu veux prendre un verre avec les collègues en fin de journée. Je connais même des types qui font leur footing sur les berges de la Garonne. C'est parfait. 
Figure 3. Spatialisation type des activités des temps de l'après travail au sein d'une centralité

Hubs comme lieu de vie : centres-villes

Maitrise de son temps / proximité

Accessibilité aux services marchands et non marchands maximale

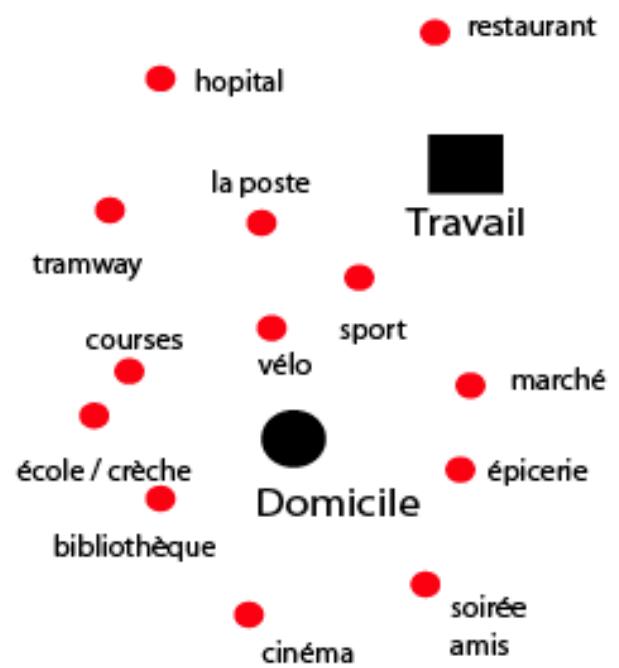

Source : J.Grosbellet, 2014

Figure 4. Spatialisation type des activités des temps de l'après travail au sein d'une centralité

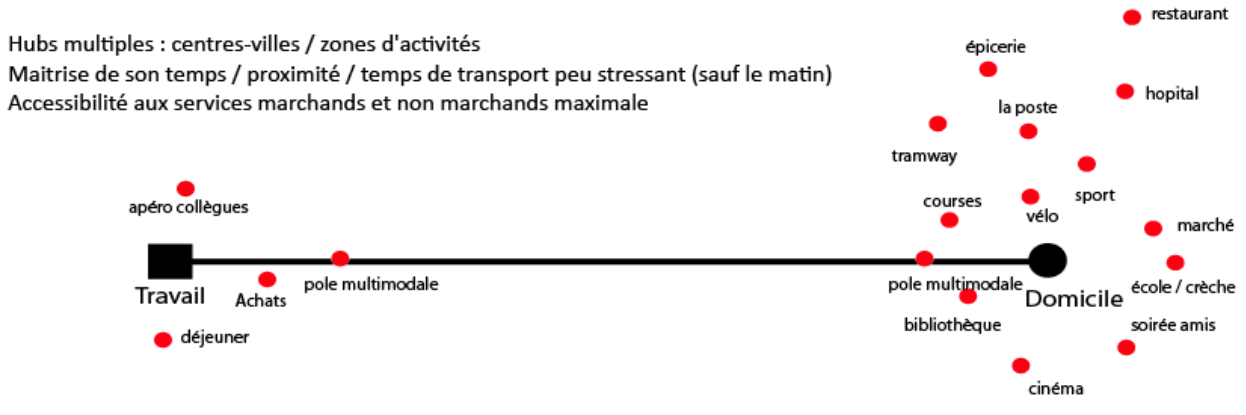

Source : J.Grosbellet, 2014

\section{Des espaces ralentisseurs pratiqués sur l'axe domicile travail}

A côté de ces parcours de "centralités », où l'axe domicile-travail n'est pas structurant, on retrouve une forme de pratique de l'espace clairement ancrée autour de ce faisceau. Après le travail, la personne a la possibilité de ressentir son temps libre « en chemin », en raccrochant son trajet à un hub. Ce parcours se retrouve souvent effectué dans l'espace périurbain, pour des personnes dont la mobilité quotidienne s'effectue dans cet espace. Les zones commerciales ou les lieux intermodaux sont le plus souvent cités. Julie qui travaille à Mérignac et vit à Gradignan considère son espace vécu quotidien autour de cet axe. Pour ces courses, elle s'arrête dans la zone commerciale de Pessac-Bersol et en 
profite parfois pour diner avec des amis dans les nombreux restaurants du site, en passant à proximité du campus, elle s'arrête faire du sport :

Bersol, c'est vraiment un endroit où je m'arrête très souvent. C'est sur mon chemin et il y a plein de choses à faire, je m'arrête aussi sur le campus pour courir certains soirs, il y a pas mal de monde. Même pour sortir ou faire les magasins, c'est plus pratique que d'aller en ville, on peut se garer. Après c'est sûr que les amis qui vivent à Bordeaux viennent rarement en banlieue. Je fais l'effort de temps en temps après le boulot mais ça s'organise.

Figure 5. Spatialisation type des activités des temps de l'après travail autour de l'axe domiciletravail

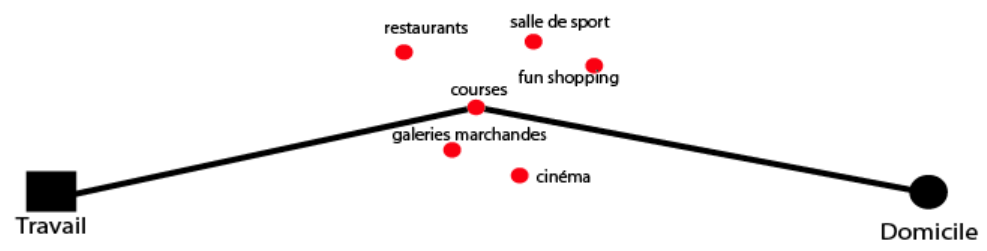

Hubs comme lieux de passage régulier : axe domicile-travail / zones commerciales / pôles multimodaux Maitrise de son temps au sein de l'axe / sas de synchronisation sur le parcours quotidien Accessibilité aux services marchands / Services non marchands peuvent nécessiter de sortir de l'axe domicile-travail

Source : J. Grosbellet, 2014

\section{Didier vit à Bordeaux, pret
sud de l'agglomération :}

Quand je sors du boulot, c'est plus cool, l'été je fais même un crochet par la plage avant de rentrer. C'est pour ça que je prends souvent ma voiture, le matin le train ça me saoule, il faut deux fois plus de temps et si j'ai des courses à faire, comme il y a un supermarché sur la route, c'est plus pratique. Le soir par contre ça m'arrive de rentrer en train, quand avec des collègues au va boire des coups à la gare de Marcheprime à côté de la boîte. Du coup le lendemain je récupère ma voiture ou je l'ai laissée.

L'espace-temps domicile-travail est aussi appréhendée comme un sas de décompression. C'est pour cette raison que Hélène a choisi de vivre à Nérigean, à 35 kilomètres de Bordeaux :

Je suis bordelaise en journée, les soirs et week-end je suis au milieu des vignes. J'ai besoin de mettre de la distance entre mon lieu de vie et mon boulot, ça me permet vraiment de couper. Je pense que c'est une chance d'avoir deux lieux de vie si différents et complémentaires.

\section{Vivre sur un fil, un parcours quotidien contraint aux rythmes discontinus}

Un troisième parcours type ressort de l'étude et concerne les personnes qui ont des emplois du temps très contraints, avec peu de marges de manœuvre et qui optimisent leurs déplacements au maximum en suivant un parcours prédéfini et optimisé. Ils sont souvent seuls dans ce parcours. L'éloignement entre leur domicile et leur travail explique en partie la contrainte du temps de transport. 
Figure 6. Spatialisation type des activités des temps contraints de l'après travail

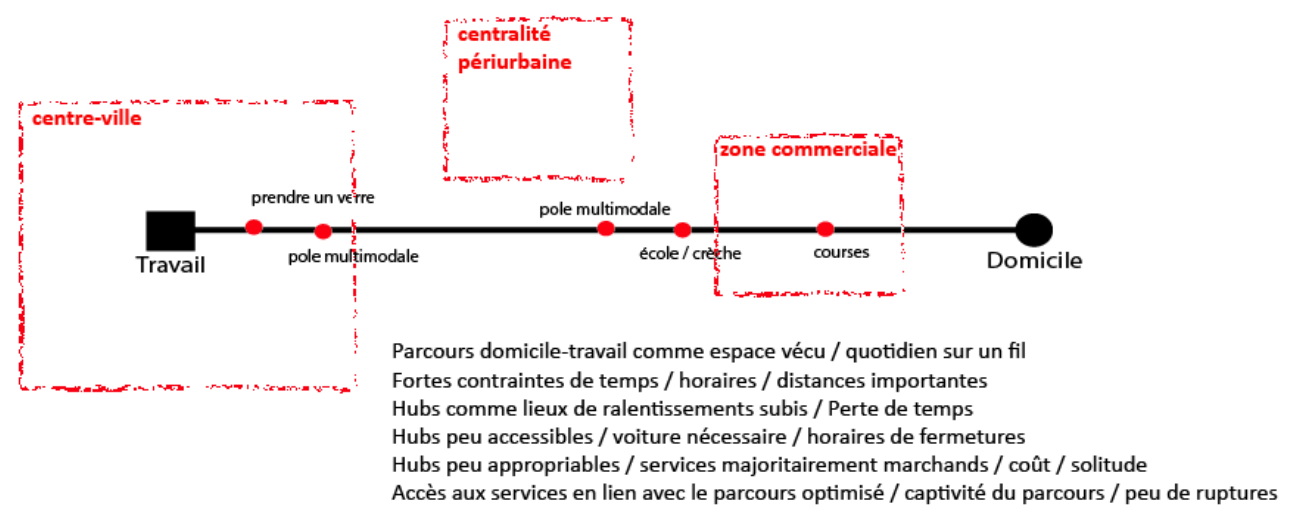

Source : J. Grosbellet, 2014

Pourtant, de la même manière que pour les parcours en faisceau, les individus croisent ou traversent des hubs. Il y a donc d'autres facteurs qui expliquent l'impossibilité pour les personnes de quitter leur «fil» quotidien. Il y a notamment le facteur économique comme le montre Pauline :

La gare de Bordeaux le matin, ça va, je ne m'y attarde pas. Parfois je prends un café avant d'aller bosser. C'est en fin d'après-midi que c'est l'enfer, ou pire le soir si j'ai eu le malheur de traîner un peu, il n'y a rien à faire à la gare à part consommer. C'est devenu une galerie commerciale. Perso, je n'ai ni les moyens ni l'envie d'acheter des souvenirs de Bordeaux tous les soirs! C'est dingue, pas de parcs, de bibliothèques... ! et la cerise sur le gâteau, c'est que souvent le train est bondé... et je dois encore récupérer ma voiture, passer retirer un colis, faire des courses...

Julie partage ce constat :

je ne peux pas prendre le risque d'aller en ville sans but précis... flâner, c'est le nouveau mot à la mode pour éviter de dire consommer en se promenant...

Pour Georg Simmel, la perception de la ville comme un lieu de consommation a détruit les temps sociaux (Simmel, 1900). Le consommateur est un usager alors que l'habitant est un acteur de la vie urbaine (Goffman, 1973). Mais au-delà des barrières économiques, c'est la linéarité du parcours qui paraît aliénante. Il y a un ordre dans l'enchaînement des temps et des espaces. Cet ordre est à la fois rassurant, car il permet la maîtrise, mais il génère également de fortes amplitudes de vitesses dans le rythme quotidien. Le cas de Fanny est particulièrement intéressant : alors que son emploi du temps est très contraint, la pause déjeuner est ressentie comme une vraie bulle d'air :

C'est la course le matin et le soir. La journée ça va. Je dépose mes enfants chez leur nounou vers $7 \mathrm{~h}$, ensuite je file pour être à $8 \mathrm{~h}$ au boulot. J'ai la chance de travailler à côté du Carrefour de Lormont, je m'y gare le matin et comme ça je fais mes courses à la pause de midi. Quand il fait beau, avec les autres employés, on va au Carrefour acheter de quoi pique-niquer et on prend la voiture pour aller un peu plus loin, au parc de l'Ermitage, c'est vraiment super. Le soir c'est reparti, il ne faut pas perdre de temps, il y a souvent des bouchons et j'ai mon deuxième travail de maman à assurer.

Il n'est pas rare de voir des temps d'accélération succéder à des temps de ralentissement subis. C'est pour cela que tous les interrogés de ce groupe, qui ont pourtant des temps libres, ne les dissocient pas toujours de leurs parcours et cela jusqu'au retour au domicile. Sophie qui est étudiante à Bordeaux et vit à Biganos, montre bien l'incertitude de l'organisation de son temps et la linéarité de son parcours : 
Après les cours j'ai souvent un peu de temps, mais ça dépend de l'heure de mon train. Des fois j'ai une heure à attendre, des fois il faut que je me dépêche. De toute façon si je veux rester boire un coup avec mes amis, je préfère venir en voiture, comme ça pas de pression. Parce qu'en plus sur le campus, il n'y a pas de bars, donc il faut aller en ville. L'été c'est plus cool, on peut squatter sur les pelouses après les cours.

En sortant du travail, Romano a un rituel qui consiste à aller prendre un verre seul. Dans ce cas, il est intéressant d'observer que pour limiter l'incertitude du futur (vais-je pouvoir prendre un verre avec quelqu'un avant de rentrer ?), Romano s'est construit un rituel, un parcours qui sécurise sa recherche d'interactions. Pour équilibrer le manque de maitrise de son temps, il s'est construit un rituel socio-spatial bien rodé :

Cela dépend des soirs mais en général, il y a toujours quelqu'un que je connais au bar. J'ai un train à prendre et le fait d'aller toujours au même bar, cela m'assure un moment de discussion avant de retrouver ma solitude dans ma campagne.

\section{Maîtriser les rythmes quotidiens : prérequis pour ralentir et réguler sa vitesse, enjeu du droit à la ville}

\section{Vers des hubs métropolitains, portes d'accès aux temps et rythmes multi-vitesses des vies intenses et mobiles}

Pour tous les enquêtés, équilibrer son emploi du temps passe par la recherche de synchronisation avec des temps et des lieux collectifs qui semblent constituer des temporalités régulatrices et structurantes. "La vie sociale s'écoule dans des temps multiples, toujours divergents, souvent contradictoires, et dont l'unification relative liée à une hiérarchisation souvent précaire, représente un problème pour toute société» (Gurvitch, 1950). Les mécanismes de ralentissement collectifs sont à réintroduire car les dynamiques individuelles peuvent se révéler discriminantes. Les développements à apporter aux espaces ralentisseurs quotidiens ne sont pas seulement de l'ordre de l'accessibilité physique, il semble que la problématique de l'appropriation soit centrale. La capacité d'appropriation est l'outil de fabrication indispensable de la ville par ses habitants. C'est la forme visible de la revendication du droit à la ville (Lefebvre, 1968). Ces espaces doivent devenir des espaces publics métropolitains dans toute leur complexité et leur diversité pour que chacun y trouve sa place. Ils doivent, au regard de cette étude, être des points de rupture dans les parcours quotidiens aux rythmes linéaires. Ils doivent s'adapter aux différentes vitesses des modes de vie, proposer des services non marchands, être connectés au reste de la ville (information en temps réel, réactivité, accès à distance) et doivent tout particulièrement replacer au centre de leur conception et de leur fonctionnement les rythmes collectifs et naturels de la lenteur.

L'aménagement de ces espaces ressort comme un enjeu primordial dans la possibilité donnée à chaque usager de rompre avec la linéarité aliénante de son parcours quotidien. Ils sont les lieux d'accès et de synchronisation à la multiplicité des rythmes de la vie urbaine. Ils jouent donc un rôle déterminant dans la maîtrise individuelle de son emploi du temps et dans le sentiment de ralentissement. Pourtant une partie des habitants de la métropole ne se synchronise pas à ces espaces. Ils en ont l'accès physique, certains les traversent, les pratiquent, mais ne se les approprient pas. Les lieux identifiés dans l'enquête, au-delà des centres-villes, sont le plus souvent des centralités périphériques mono-fonctionnelles et mono-vitesse, celles de la rapidité et de la consommation. 
Pourtant, l'usage de ces non-lieux (Augé, 1992) a contribué, avec le temps, à les façonner en lieux de vie quotidienne pour de nombreux habitants. Toutefois, il manque encore des aménagements importants pour que ces espaces soient appropriés comme des centralités urbaines. La proximité de services marchands et non marchands, les espaces publics, la diversité des modes de déplacement, la vie sociale et culturelle sont autant d'éléments qui permettent à des habitants aux rythmes et modes de vie différents de partager un même espace. Ces lieux doivent également pouvoir faire cohabiter rapidité et lenteur, car la ville se pratique à différentes vitesses, à différents moments. Elle est le lieu par excellence où se confrontent des groupes sociaux multiples possédant chacun des temporalités qui leur sont propres mais organisées en activités collectives (Eberling, 1997). Mais si l'espace se laisse transformer facilement par l'aménagement, le temps est plus difficile à apprivoiser (Boutinet, 1996 : 312).

\section{Les temps sociaux et naturels comme métronomes urbains : replacer les rythmes linéaires des parcours quotidiens en interaction avec les rythmes cycliques de la lenteur}

37 Le droit à la ville encourage une réappropriation collective de la ville. Il semble que la régulation des rythmes de vie passe également par la redéfinition des rythmes collectifs (temps sociaux, rythmes cycliques, cosmiques) structurant les rythmes individuels :

Cette propension à vouloir changer de lieu pour se retrouver avec d'autres ou dans la nature, dès qu'on dispose d'un peu de temps libre pourrait traduire, selon notre hypothèse, le besoin de vivre des rythmes dont la source est extérieure à soi-même et qui se différencient de ceux du travail, sans toutefois être moins intenses. (Trautman, 2007)

38 Identifiés comme des temporalités structurantes au sein du milieu urbain, les temps sociaux et les temps naturels régulent les rythmes quotidiens des individus. L'espace fonctionne "[...] toujours en interaction constante avec un ou plusieurs groupes sociaux" (Tomas, 2003) et ceux-ci induisent des temporalités qui leurs sont propres. Bien que ces rythmes soient singuliers, ils s'organisent en fonction de repères et de rythmes qui sont comparables à ceux des temps sociaux (Chesneaux, 2004). En se synchronisant avec ces temps collectifs, l'individu unifie sont temps personnel avec celui d'une globalité rassurante. Ces rythmes collectifs sont à la fois sécurité, habitudes et invention, enrichissement inattendu (Godard, 2003). Lieu privilégié de l'accomplissement des temps sociaux, l'espace public est l'espace collectif où s'expriment et se croisent des rythmes pluriels. Mais d'après un paradoxe qui semble s'imposer, "si les temps sociaux sont fondamentalement pluriels, la société ne peut vivre sans essayer d'unifier cette pluralité» (Mallet, 2013).

39 L'enjeu semble donc de marquer les temps sociaux et naturels, repères et témoins d'un mode de vie partagé afin que chaque individu puisse s'y référer. Ils agissent ainsi en métronomes de la vie quotidienne. Hélène $a$ :

aussi la possibilité de dormir chez des amis sur Bordeaux, l'hiver surtout, quand

c'est un peu difficile de rentrer de nuit. L'été ce sont plutôt mes amis qui viennent.

Que l'on vive en rythme ou à contre-temps, les temps naturels comme l'alternance journuit permettent de se positionner. Simon, qui travaille en horaires décalés, est particulièrement sensible au décalage entre temps de travail et temps naturels : 
Franchement, depuis que je bosse avec ces horaires (4h30-11H30), je ressens vraiment le changement entre les saisons. Avec le lever et le coucher du soleil notamment. L'été, je prends des trucs pour dormir, il faut que je garde le même rythme mais c'est pas facile, j'ai vraiment tendance à me coucher plus tard sinon.

41 En ce qui concerne les temps naturels, ils permettent de replacer l'homme en interaction avec son biorythme. C'est notamment le cas des jardins urbains qui réintroduisent des temps naturels en ville. Pour Blair, qui possède un jardin privatif à l'arrière de sa maison :

Il me permet de ralentir mon rythme quotidien en introduisant une sorte de fil rouge tout au long de l'année, cela me fait relativiser plein de choses, et notamment de me retrouver au chômage ou au RSA... car je n'y connaissais rien et je me rends compte avec le temps que je suis maintenant capable de faire pousser des légumes.

Plus généralement, tous les enquêtés indiquent que la recherche de vie sociale et le changement de climat et/ou de saisons influent sur leur capacité à sortir de leur routine quotidienne. "Prendre avec verre avec des amis ", « la pause déjeuner » sont des temps de ralentissement souvent cités comme choisis. «L'été » invite plus au ralentissement que «l'hiver », il faut « profiter du beau temps pour sortir ». Dans son étude de rythmanalyse, Henri Lefebvre distingue les villes solaires et les villes lunaires. Les villes solaires se trouvent sur la façade méditerranéenne car rythmées par la course du soleil. Les modes de vie s'adaptent aux fortes chaleurs, le travail aussi. Les villes lunaires sont rythmées par la marée, ce sont des villes océaniques, portuaires, où la marée joue un rôle prépondérant dans l'organisation du rythme de la vie urbaine. Il insiste également sur l'importance de l'étude des interactions entre les rythmes linéaires et aliénants du capitalisme et les rythmes cycliques et structurants de la vie sociale et de la nature (Lefebvre, 1992).

\section{Conclusion}

La maitrise des rythmes quotidiens semble s'imposer comme une nouvelle revendication du droit à la ville. Elle s'appuie sur deux éléments en particulier. D'une part, elle requiert pour chaque individu, la possibilité de mieux maîtriser son temps et ainsi avoir le sentiment de ralentir. Le temps ne doit plus seulement être perçu comme un capital mais bien comme une liberté individuelle, celle de choisir. En ce sens, l'aménagement d'espaces ralentisseurs, comme hubs métropolitains au sein du parcours quotidien, faisant cohabiter rapidité et lenteur, temps personnel et temps collectifs, temps marchands et non marchands, semble être une première étape pour permettre au plus grand nombre de s'approprier et de se synchroniser aux échelles et rythmes de vie quotidiens. Ces espaces jouent un rôle structurant dans la régulation et la maîtrise des temps quotidiens, c'est-à-dire des espaces dont la connaissance, la compréhension et l'aménagement représentent un enjeu important non seulement en ce qui concerne l'accès à la ville elle-même mais aussi l'accès à ses usages, autrement dit à ses temporalités. Cela passe également par une amélioration de l'appropriation de ces espaces-temps singuliers (l'after-work, la pause déjeuner, les transports, les lieux de connexion intermodales, les zones économiques et commerciales, les centralités urbaines de manière général), et par le développement et la diffusion d'outils numériques permettant de mieux gérer son parcours quotidien en temps réel.

D'autre part, il semble important d'accorder une attention toute particulière à la régulation des rythmes de la vie quotidienne à travers leur ancrage aux temps collectifs et naturels, exogènes et structurants. L'apparition d'un «capital temporel » témoigne 
bien de la pénétration des rythmes du capitalisme dans les modes de vie entraînant une dévalorisation des temps collectifs au profit d'une logique individualiste de l'organisation de son emploi du temps. Dans notre cas d'étude, un emploi du temps subi et contraint a tendance à montrer une prédominance des rythmes linéaires des parcours quotidiens sur les temps sociaux et naturels. Les temps quotidiens maîtrisés semblent prendre place dans une centralité urbaine et sociale structurante. «Le rythme individuel est entraîné par celui de la foule, celle-ci est un donneur de temps qui synchronise ses membres. » (Lasén, 2001). Il faut alors continuer à favoriser des scansions rythmiques collectives qui dessinent la forme propre d'une ville et porter attention aux possibilités de favoriser leur expression (Michon, 2005). En sortant un peu du cadre de l'étude, nous pouvons identifier le weekend et plus particulièrement le dimanche comme relevant d'une temporalité sociale structurante. Si les capacités d'accélération et de ralentissement diffèrent selon chaque personne, la réintroduction des temps cycliques de la lenteur, comme repères et métronomes des modes de vie métropolitains, est devenu un enjeu majeur de la revendication du droit à la ville.

\section{BIBLIOGRAPHIE}

ASCHER F. (1997) « Demain, la ville de tous les temps » in OBADIA A. (dir.), Entreprendre la ville, Nouvelles temporalités-Nouveaux services, Colloque de Cerisy, La Tour d'Aigues, éditions de l'Aube, pp. 17-32.

AUGUSTIN (Saint) (1962), Les confessions, Livre XI.

BARRERE-MAURISSON M-A., RIVIER S. (2004) « Partage des tâches et des temps dans les ménages et parité professionnelle », in ISTACE E., LAFFUT M., PLASMAN R., RUYTERS C., Sphères privée et professionnelle, vers une recomposition des rôles et des actions, De Boeck supérieur, pp. 295-311.

BENVENISTE E. (1966), Problèmes de linguistique générale, Paris, Gallimard, 356p.

BOURDIN A. (2005), La métropole des individus, Paris, éditions de l'Aube, 249p.

BOUTINET J-P. (1996), Anthropologie du projet, Paris, PUF, 397p.

BORREL P. (2014), L'urgence de ralentir, documentaire, ARTE France, 84 minutes.

BOURDIEU P. (1979), La Distinction : critique sociale du jugement, Paris, Editions de Minuit, 670p.

CHARDONNEL S. (1999), Emplois du temps et de l'espace. Pratiques des populations d'une station touristique de montagne. Thèse de Géographie, Grenoble, Université Joseph Fourier, 230p.

CHESNEAUX J. (1996), Habiter le temps : passé, présent, futur, esquisse d'un dialogue politique, Paris, Bayard, 344p.

CHESNEAUX J. (2004) « Cinq propositions pour appréhender le temps », Temporalités, 2004/1 http://temporalites.revues.org/648, consulté le 20 août 2015.

CINGOLANI P. (2012), Le Temps fractionné. Multiactivité et création de soi, Paris, Armand Colin, 220p. 
COSTES L. (2014) « Néolibéralisation et évolution du 'Droit à la ville' « , Justice Spatiale / Spatial Justice, 2014/6

http://www.jssj.org/article/neoliberalisation-et-evolution-du-droit-a-la-ville/, consulté le 20 août 2015.

COULANGEON P., MENGER P-M., ROHARIK I. (2012) « Les loisirs des actifs : un reflet de la stratification sociale », Economie et statistique, $\mathrm{n}^{\circ}$ 352-353, pp39-55.

DEBORD G. (1967), La société du spectacle, Paris, Gallimard, 224p.

DUBAR C. (2008) « Temporalité, temporalités : philosophie et sciences sociales », Temporalités, $2008 / 8$

https://temporalites.revues.org/137, consulté le 20 août 2015.

EBERLING M. (1997) « Une politique des horaires urbains » in OBADIA A. (dir.), Entreprendre la ville, Nouvelles temporalités-Nouveaux services, Colloque de Cerisy, La Tour d'Aigues, éditions de l'Aube, pp. 138-143.

FREMONT A. (1999), La région, espace vécu, (1972 pour la première édition), Paris, Flammarion, $288 p$.

GODARD F. (2003) « Cessons d'opposer temps individuels et temps collectifs », Projet, 2003/1 (n -273), pp. 35-42.

GOFFMAN E. (1973), La mise en scène de la vie quotidienne, les relations en publics, Paris, éditions de Minuit, 368p.

GROSSIN W. (1996), Pour une science des temps : introduction à l'écologie temporelle, Toulouse, Octares, 268p.

GURVITCH G. (1950), La vocation actuelle de la sociologie, Paris, PUF, 507p.

HAGERSTRAND T. (1970) «What about people in regional science? », Papers of the Regional Science Association, no. 24, pp. 7-21.

HONORE C. (2005), Eloge de la lenteur, Paris, Marabout, 287p.

KAUFMANN J-C. (1997), Le cœur à l'ouvrage, théorie de l'action ménagère, Paris, Nathan, 238p.

LASEN A. (2001), Le temps des jeunes. Rythmes, durée et virtualités, L'Harmattan, 322p.

LEFEBVRE H. (1968), Le Droit à la ville, Paris, Anthropos, 164p.

LEFEBVRE H. (1992), Eléments de rythmanalyse : introduction à la connaissance des rythmes, Syllepse, Paris, $122 p$.

MALLET S. (2013) « Aménager les rythmes : politiques temporelles et urbanisme », EspacesTemps.net, Laboratoire, 15 avril 2013.

http://www.espacestemps.net/articles/amenager-les-rythmes-politiques-temporelles-eturbanisme/, consulté le 20 août 2015.

MARZLOFF B. (2005) « Les nouvelles temporalités de la ville », POUR, numéro 188, 12/2005, pp. 164-168.

TOMAS F. (2003), Les temporalités des villes, Saint-Etienne, Publications de l'Université de SaintEtienne, 219p.

PERCHERON A. (1988) «Classes d'âge en question », Revue française de science politique, 1988/1, pp. 107-124.

ROSA H. (2010), Accélération. Une critique sociale du temps, Paris, La Découverte, 474p. 
SIMMEL G. (1987), Philosophie de l'argent, (1900 pour la première édition), Paris, PUF, 662p.

VIRILIO P. (2003), Ville panique, Ailleurs commence ici, Galilée, 144p.

\section{RÉSUMÉS}

"Ralentir ", " prendre son temps ", " avoir plus de temps », sont autant de souhaits exprimés quasi-unanimement lors d'une enquête sociologique menée auprès de dix-huit personnes qui vivent et/ou travaillent dans la métropole bordelaise. Pourtant, en spatialisant et en observant les parcours quotidiens de chaque individu, il existe de nombreux temps et espaces ralentisseurs qui viennent rythmer leur journée. Les temps de transport, d'attente, les gares ou lieux multimodaux, les zones commerciales ou d'activités, ne semblent toutefois pas être vécus de la même manière par les personnes interrogées. Certains aimeraient accélérer et ne pas subir cette lenteur à cet endroit et/ou à ce moment de la journée. D'autres choisissent au contraire de profiter de cet espace-temps comme d'un lieu de synchronisation et d'équilibre dans leur parcours quotidien. L'aménagement de ces espaces et temps ralentisseurs semble alors représenter un enjeu majeur dans la maîtrise individuelle de son temps et dans la revendication plurielle du droit à la ville.

To " slow down ", " take time ", " have more time ». Here are some of the wishes expressed by almost all of the twenty people, leaving and/or working in the city of Bordeaux, that have been interviewed through a sociological investigation. However, by spatializing and observing the daily journey of each individual, it occurred that numerous times and slowing down spaces do exist, punctuating their day. Transport and waiting times, railway stations or multimodal places, commercial or working areas, do not appear to be experienced the same way by respondents. Some would like to accelerate in order not to suffer from this slowness in that place and / or at that time of the day. Others choose instead to take advantage of this space-time as a place of synchronization and balance in their daily journey. The planning of these slowing down spaces and times then appears to be a major issue in the individual control of one's time and in the plural claim of a right to the city.

INDEX

Thèmes : Carnets de recherches

\section{AUTEUR}

\section{JEAN GROSBELLET}

